\title{
Numerical Study of Low Reynolds Number Flapping Wing Da-Min CAO ${ }^{1, a}$, Hong-Yan LV1', Lu-Hui YANG ${ }^{1}$
}

1School of Air Transportation / Flying, Shanghai University of Engineering Science, 201620, China

Keywords: Flapping wing, Hovering insect, Low Reynolds, Unsteady lift mechanism.

\begin{abstract}
The influence of different flapping wing models on the aerodynamic performance of a hovering insect is numerically investigated. Some simplified models are compared with the aerodynamic performance of the hovering fruit fly wing. With the increase in the complexity, two different models are considered and dynamically scaled at Low Reynolds number (about 110). The three-dimensional movement of the fruit fly hovering flapping wing was simplified to the twodimensional motion. The numerical results capture the stall delay effect, and the fast rotation force effect of the unsteady lift mechanism. The lift and drag coefficient curves by two kinds of flapping model are compared.
\end{abstract}

\section{Introduction}

MAV use the flapping wings to produce enough thrust and lift for flapping up and down, and back and forth. Referring to the bionics research of the insects and birds [1-6], the wing combines the rotation, misalignment up and down, and movement back and forth. Consequently, the aircraft can advance forward and backward, hover and lift. In addition, several investigations show that for the bionics [7], when the wing length is less than a certain value (about $15 \mathrm{~cm}$ ), the aerodynamic lift generated by the flapping wing is far higher than the fixed-wing, resulting in a higher propulsion efficiency.

\section{Numerical Simulation Methods}

\section{Dickinson Robofly Models}

In this paper, the Dickinson [6] and Robofly wing models are selected. The cross section of the spanwise positions which can fully reflect the aerodynamic characteristics of the three-dimensional insect wing model is simulated, as shown in figure 1 . The wing is $0.254 \mathrm{~m}$ in the spanwise direction, and the area, $\mathrm{S}$, is $0.0167 \mathrm{~m} 2$, and the airfoil section of $\mathrm{Rg}$ is $0.6396 \mathrm{R}$. The airfoil is a short shaft with a long axis length of $2 \%$ elliptical thin, the section chord $\mathrm{C} 0.114 \mathrm{~cm}$. The reference length, namely the average chord length, is calculated to be $0.082 \mathrm{~cm}$ by using the method of moment of inertia, and the rotating centre is based on the root, as shown in figure 2.

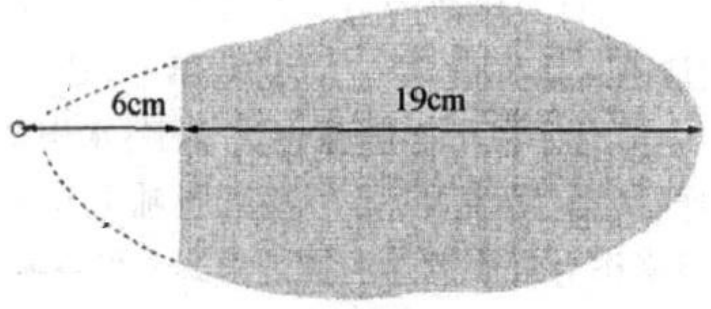

Figure 1. wing model

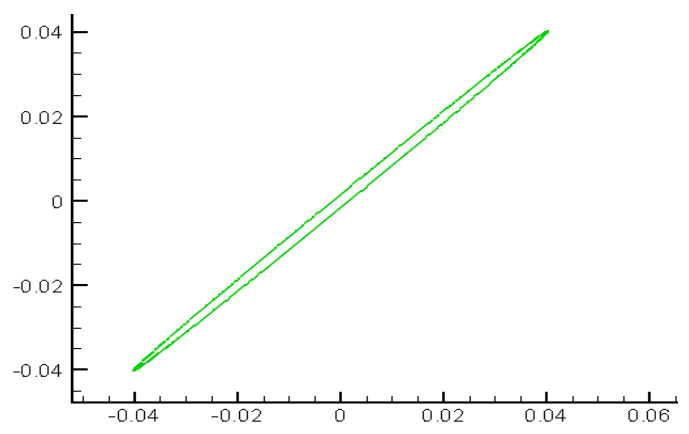

Figure 2. Dickinson Robofly model

\section{Grid and Boundary}

The flow field around the two-dimensional airfoil is calculated by the $\mathrm{O}$ type calculation domain, as shown in Figure 3. The grid is divided into two computational domains, O1 and O2. The airfoil 
surface layer F1 is located at the centre of the computational domain. F2 is located in the inner grid boundary of O1. The length of computational domain is 15 times than the airfoil chord; F3 is outside the $\mathrm{O} 2$ grid boundary, whose computational domain is 15-20 times than the airfoil chord. Considering the hovering flight state of the fruit fly, F3 uses the symmetry boundary conditions. In order to simulate the trailing edge vortex generated by both ends of the airfoil, the number of the grid around the airfoil is much higher. A two-dimensional thin airfoil was used. The small curvature radius of the airfoil trailing edge requires very dense mesh. The middle airfoil geometry gradient is very small, and the grid is relatively sparse. Therefore, the number of F1 grid at the middle of the airfoil is about $50 \%$ of the thickness, and the airfoil is about $5 \%$ of the trailing edge of the airfoil thickness of the mesh size, as shown in figure 4.
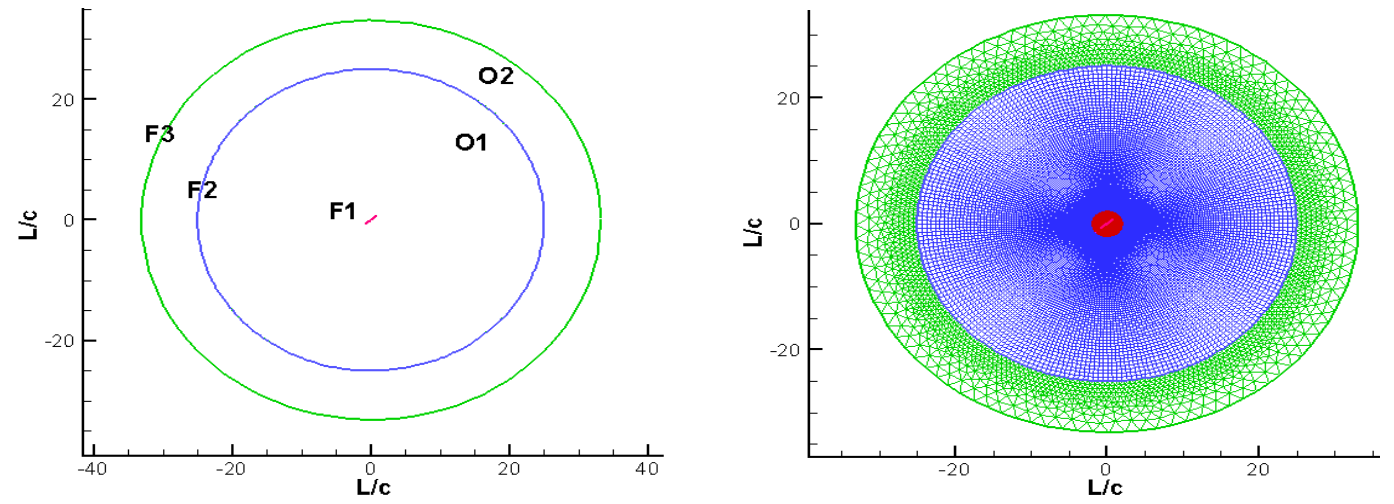

Figure 3. Computation domain(Green boundary is F3, blue boundary is F2 and red boundary is F1)

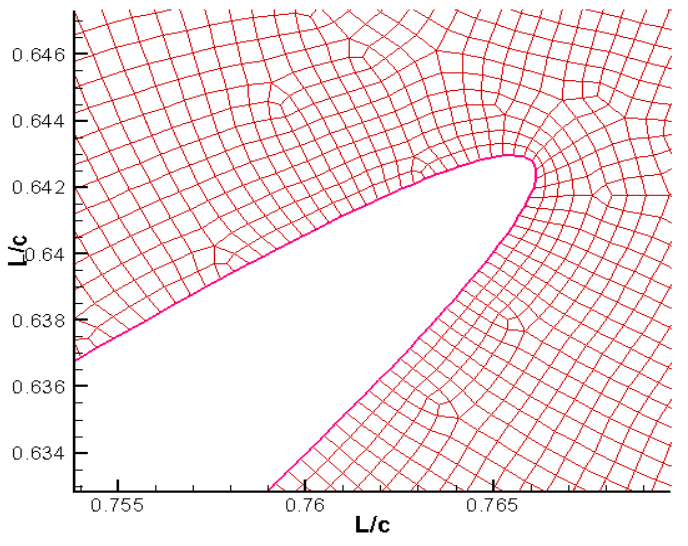

(a)Aerofoil leading edge

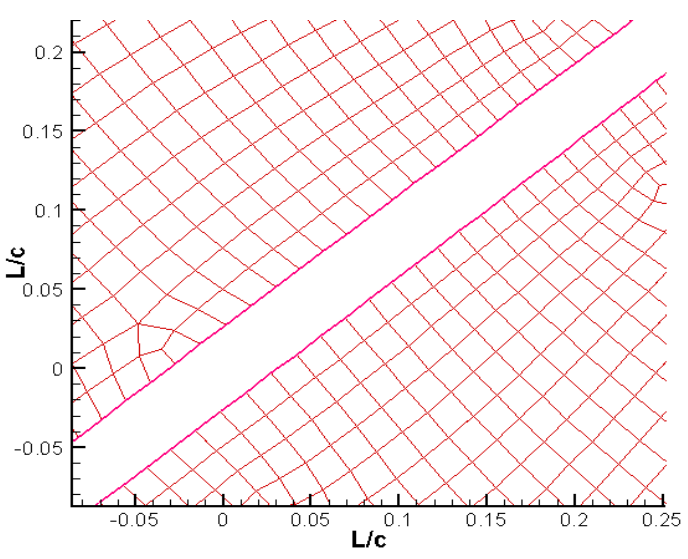

(b) Aerofoil middle section

Figure 4. Airfoil surface meshing

\section{Motion Model}

The three-dimensional motion of the flapping wing model has three degrees of motion, namely the flapping angle, deviation angle and angle of attack. If the angle of attack is not taken into account, the airfoil cross section at any point of the trajectory of the space in the wing root as the centre of the circular path. Three dimensions can be simplified as the two-dimensional motion of dimensionless equations:

$$
\bar{x}=\frac{\phi R_{g}}{\bar{c}}, \bar{y}=\frac{\theta R_{g}}{\bar{c}}
$$

The circular path around the flapping up and down movement of the shift was converted into the linear displacement of the 2D airfoil cross-section along $\mathrm{X}$-axis and $\mathrm{Y}$-axis direction. Here $\phi R_{g}$ and $\theta R_{g}$ were the length of the flapping motion and drift motion in space through a circular path. The angle of attack of the three-dimensional model is transformed into the rotational motion 
around the airfoil aerodynamic centre. The airfoil is located in the rotation centre, a quarter of the average chord length.

Figure 5 shows a schematic diagram of the two-dimensional motion model system. $\mathrm{X}$-axis corresponds to the average flapping plane. The upwards of the Y-axis and the left forward of the Xaxis are specified to be positive. In the figure, the leftmost is the starting position of the upward flapping, the rightmost is the starting position of the downward flapping, the $2 \mathrm{D}$ airfoil along the Xaxis from the leftmost to the rightmost of flapping is a upward trip, from the rightmost along the Xaxis to the leftmost is the downward trip, and the middle of this is the coordinate origin. In a flapping cycle, the wing of the leftmost starting to do the half a rightward cycle motion, flapping change direction after reaching the extreme right, and then left to do the next half-cycle beat movement, reach the extreme left. The displacement along the $\mathrm{X}$-axis and $\mathrm{Y}$-axis is proportional to the flapping and deviation angles, respectively. Representing the angle of attack, the flapping angle and deviation angle, the angle of attack varies with time, or its angular velocity varies with time, we can express a complete motion model. Providing the flapping angle and deviation angle in the Xaxis and Y-axis displacement is positive, otherwise it is negative. For the attack angle, the counter clockwise rotating around the $\mathrm{X}$-axis is positive. When the wing is the starting position of upwards and downwards, the angle of attack are 90 degrees.

In this paper, the numerical simulation adopts two flapping wing models: a harmonic flapping model (as shown in Figure 6), the flapping angle and angle of attack changes over time in line with sine or cosine function; Figure 7 shows a real fruit flies model of flapping and flapping angle, angle of attack deviation angle and change over time [6]. Since the real flapping complexity of the model, any analytical equation for describing it will lose a certain authenticity. Figure 8 shows the actual simplified flapping model, which uses the flapping angle on flutter cycle symmetrical sine function representation; deviation angle change over time also reduces to about asymmetrical flutter cycle sine function there is a certain phase shift, which is to simulate the real flap asymmetry model deviates from the movement; the angle of attack in the middle stage flapping more gentle stroke, is reduced to a constant angle of attack, that is a straight line, while the other part different from the sine or cosine function can be simplified. To simulate the convenience, the angle of attack is flapping cycle symmetrical.

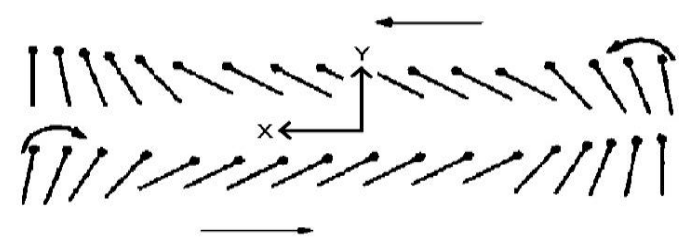

Figure 5. two dimensional motion

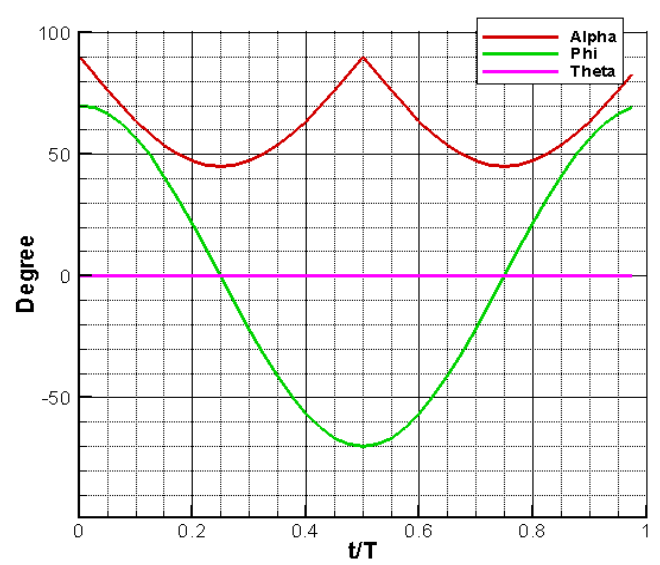

Figure 6. Harmonic model

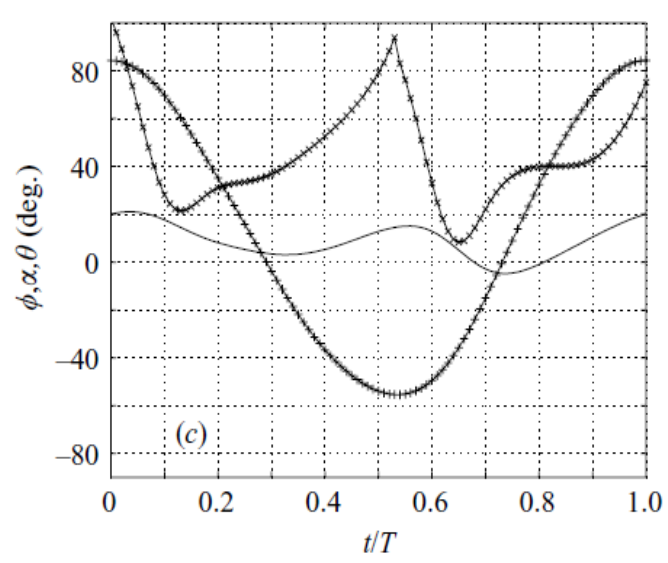

Figure 7. Real fruit fly model 


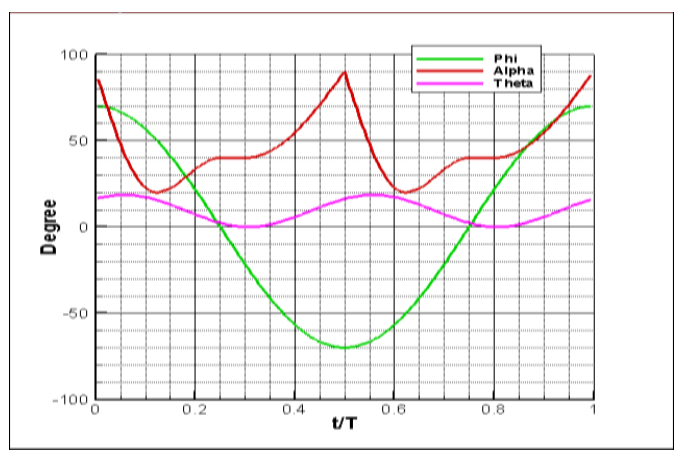

(a)Angle of movement versus time

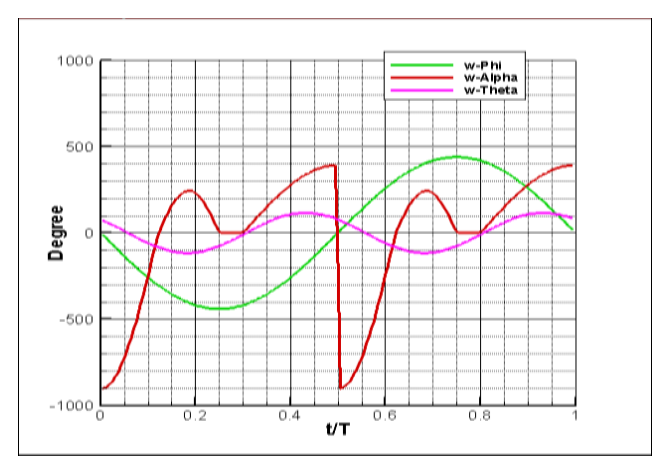

(b)Angular velocity versus time

Figure 8. Simplified model

\section{Result and Analysis}

\section{Harmonic Model}

This section flapping harmonic model and the simplified model calculates flapping 10 cycles, each cycle flapping into 200 time steps. Figure 9 shows the curves of harmonic model flapping lift coefficient and you can see lift and drag coefficients tend to change periodically after the first six cycles. Therefore, take the cycle (28.75-33.75s) for the curve lift and drag coefficients, and the dimensionless time processing, as shown in Figure10. Since the lift and drag coefficients flapping stroke (ie, before and after the half flapping cycle) changes in almost the same, indicating that the model corresponding to the flapping rule is consistent. Flapping in a stroke, there are two obvious lift lift peak, respectively, in the second half of flapping travel and after the first half, and the second peak is greater than the first peak. A flapping period average lift coefficient is 1.409 , a flapping stroke (half flapping cycle) average drag coefficient of 2.393, the average lift to drag ratio of 0.419 .

\section{Lift Coefficient Analysis}

Figure 11 shows the inner surface of the airfoil stroke flapping like vorticity contours, flapping on the itinerary can be divided into four stages

$$
\bar{t}=0 \sim 0.1
$$

When the $\mathrm{t}=0$, in the beginning of the state on the airfoil, the attack angle is 90 degrees, flapping speed is 0 . Since then, right on the airfoil motion, and clockwise rotation, the lift coefficient is rising, and reached the first peak at $\mathrm{t}=0.1$ about 1.9. Increased lift coefficient may be two reasons: one is the airfoil during acceleration due to the inertia of the fluid, so that the positive direction of the forward lower wing surface to generate high pressure, and with his back to the forward direction of the airfoil produces a relatively low pressure to produce high-lift, a phenomenon known as the added mass effect; the other was the presence of a high intensity vortex structure (shown in Figure 11a) in the forward direction of the airfoil, it fluttered on a stroke airfoil leading edge is formed, and at the time of transition from the flapping wing stroke off the leading edge, the leading edge of the airfoil after the end of exposure to the vortices, the establishment of the initial flow circulation, resulting in the lift, a phenomenon called wake capture effect. However, the leading edge of the airfoil and time of wake vortices contact does not match the peak time of the first lift. The first lift at peak time, then wake vortex strength and shape is relatively new to $0.05 \mathrm{~s}$ when already on the wane, and then wake vortices on end lift, but the first time a trough lift appearing is close to $0.2 \mathrm{~s}$. Therefore, it is considered to have limited effect on the impact of wake capture lift, lift the first major peak acceleration airfoil by flapping effects.

$$
\bar{t}=0.1 \sim 0.175
$$


This stage, the lift coefficient from 1.9 to 0.9 , including two possible reasons: 1 , the leading edge vortex does not reside on the surface of the airfoil, cannot form a high-lift; 2, due to the flapping of the acceleration is gradually reduced, coupled with a certain change the angle of attack, so fast acceleration effect of weakening, lift decline.

$$
\bar{t}=0.175 \sim 0.325
$$

This stage, the lift coefficient increased from 0.9 to 2.82 second peak lift. This stage can be divided into two parts: 1 , due to flapping acceleration continues to decrease speed gradually reduce the angle of attack, this time to do a similar airfoil angle of attack constant uniform flapping in the leading edge vortex been gradually formed, lift rise, as shown in Figure 11e and f; 2, wing flapping, while reducing speed, angle of attack accelerated change, rapidly rotating airfoil on belief, formed a rapid rotational force effect, making sharp rise lift, appeared for the first two peaks.

$$
\bar{t}=0.325 \sim 0.5
$$

This stage, the lift coefficient decreased from 2.83 to 0.1 . Due to the fast deceleration effect flapping, so that the upper wing surface pressure increases, the lower wing surface pressure drops. In addition, the angle of attack of 90 degrees continue to change, vertical airfoil gradually, not only in the leading edge of the left wing vortex, increasing the vertical area also enhances the effect of rapid deceleration, so that the lift coefficient dropped significantly.

In short, harmonic flapping model high lift coefficient is mainly constituted by the following factors: 1, flapping in the beginning, by the additional mass of the airfoil effect rapid acceleration flapping generated with appropriate changes in the angle of attack to produce a first lift peak; 2, flapping in the second half, Yang with the rapidly rotating force effect certain flapping speed generated to produce a second peak on the airfoil lift fast; 3, the leading edge vortex effect of delayed stall had some lift, but the lift is not a major factor; 4, wake capture effect may have some lift upgrade, but the effect is not obvious.

\section{Drag Coefficient Analysis}

Aircraft resistance mainly comes from the direction of the pressure before and after flapping airfoil. Greater lift, upper and lower surfaces of the pressure difference the greater the pressure difference before and after the corresponding airfoil greater, resulting in greater resistance, therefore, there is a certain correspondence between the drag coefficient and lift coefficient. In one stroke flapping, drag coefficient, there have been two peaks, and the peak time of the lift coefficient is substantially the same.

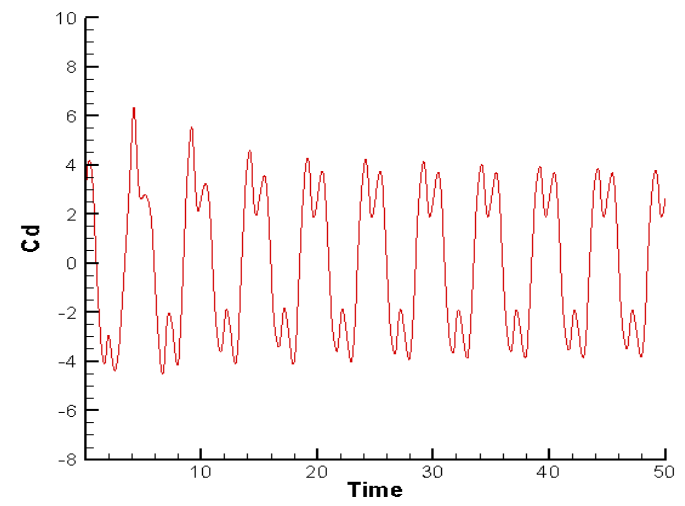

(a)lift coefficients

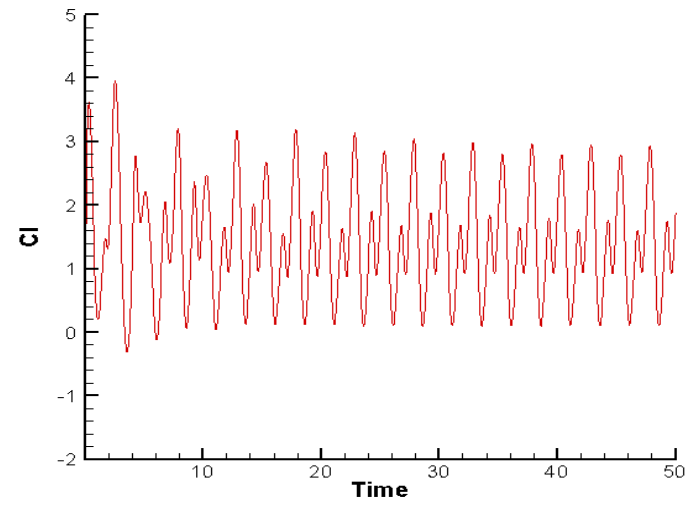

(b) drag coefficients

Figure 9. lift, drag coefficient curve of harmonic model 


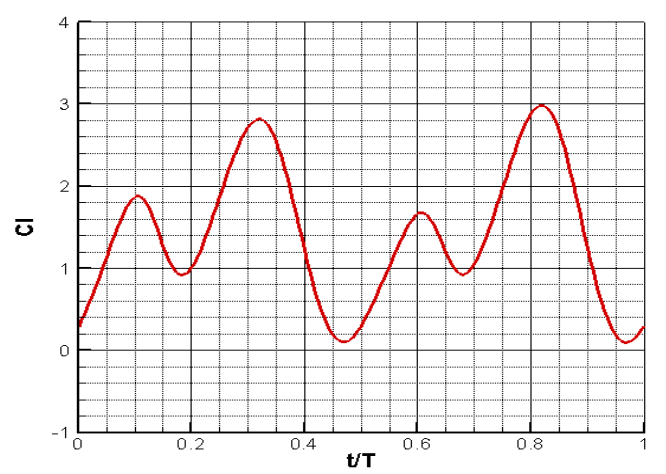

(a)lift coefficients

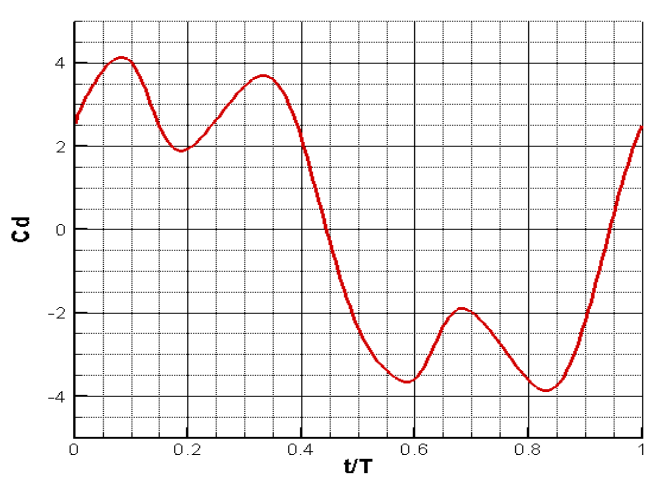

(b) drag coefficients

Figure 10. lift, drag coefficient curve of harmonic model flapping cycle

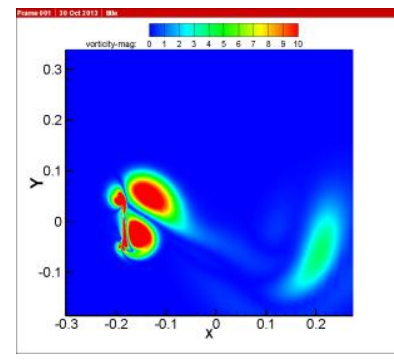

(a) ${ }_{t}^{-}=0$

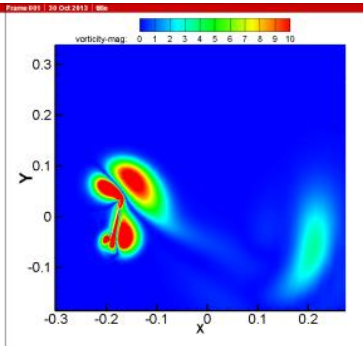

(b) ${ }_{t}^{-}=0.05$

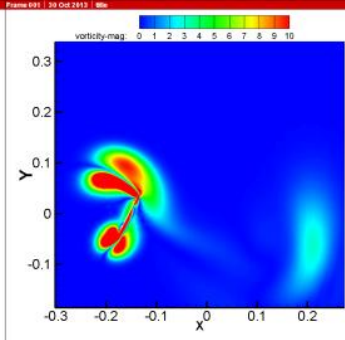

(c) ${ }_{t}^{-}=0.1$

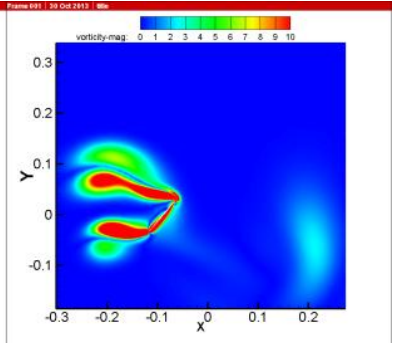

(d) ${ }_{t}=0.175$

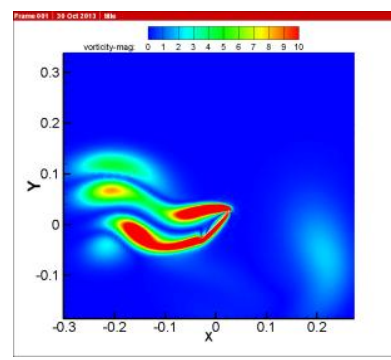

(e) $\bar{t}=0.25$

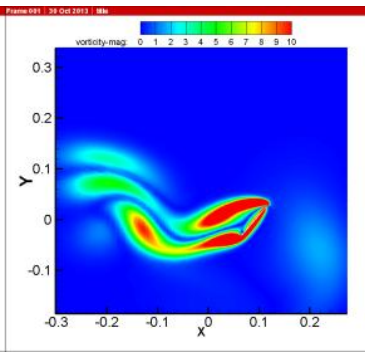

(f) ${ }_{t}^{-}=0.325$

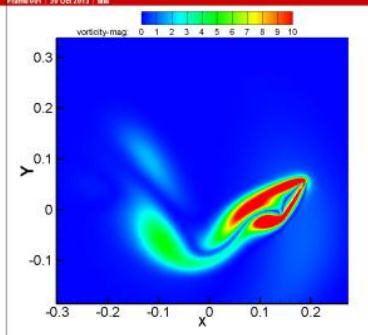

$(\mathrm{g})_{t}^{-}=0.4$

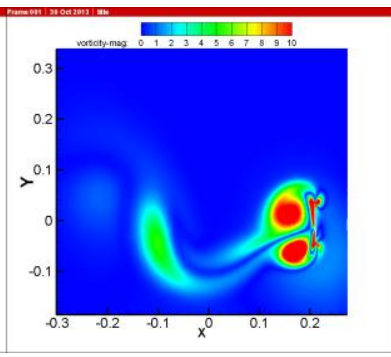

(h) ${ }_{t}^{-}=0.5$

Figure 11. Vorticity contour of upward flapping itineraries of the harmonic model

\section{Simplified Fruit Fly Model}

Figure 12 shows a simplified model in a flapping flapping cycle (ie between 29.88-35.25s) lift and drag coefficients maps and dimensionless time processing. The average lift coefficient of a flapping stroke (half flapping period) is 1.71 , the average drag coefficient of 1.83 , the average lift to drag ratio of 0.765 , than harmonic flapping model lift to drag ratio increased roughly $82.6 \%$. It is noteworthy that, in flapping stroke conversion stage, there has been a mutation lift coefficient. This mutation is made in the upper and lower beat stroke conversion phase attack occurred due to the angular velocity of the mutation.

Simplified models produced lift flapping mechanism is more complex, in addition to the effects of rapid rotational force added mass effect flapping angular acceleration fast movement of the leading edge delay and stall vortex effect in airfoil caused rapid elevation, its deviation from the vertical movement and the angle of attack curve downward "extra uplift" changes also have some impact on the generation of lift. Effect of deviation from the vertical movement of the lift coefficient mainly as follows: when the wing flapping down from the top, the added mass effects of fluid inertia caused by such lift coefficient increased significantly, and in the airfoil from flapping upward, additional mass effect but also makes the airfoil lift coefficient has been reduced, shown in Figure 12a. On flapping trip, departing from the downward motion, flapping down stroke on the 
contrary, so the flapping stroke lift coefficient significantly higher than under the flapping stroke. Downward angle of attack "extra bump" airfoil change makes the next stoop to the lowest value in a short time and then 20 degrees to 40 degrees elevation, suitable flapping movement of the mating, resulting in a rapid rotational force effect, making the lift coefficient obviously increase. This can be seen between the highest lift of Figure 12a produced by the peak seen. In addition, 13a and b can be seen in this "bump down" phase and the subsequent movement phase constant angle of attack, the wing front edge of the airfoil produces the intensities of the two largest models leading edge vortex, which is the angle of attack "extra uplift" changes are closely linked.

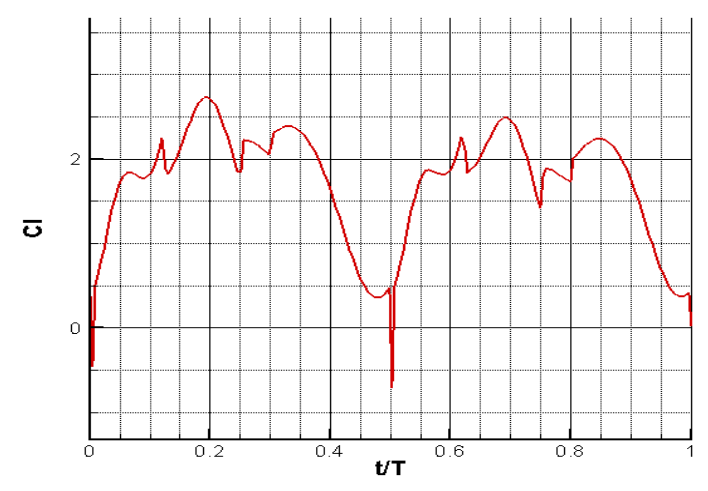

(a)lift coefficients

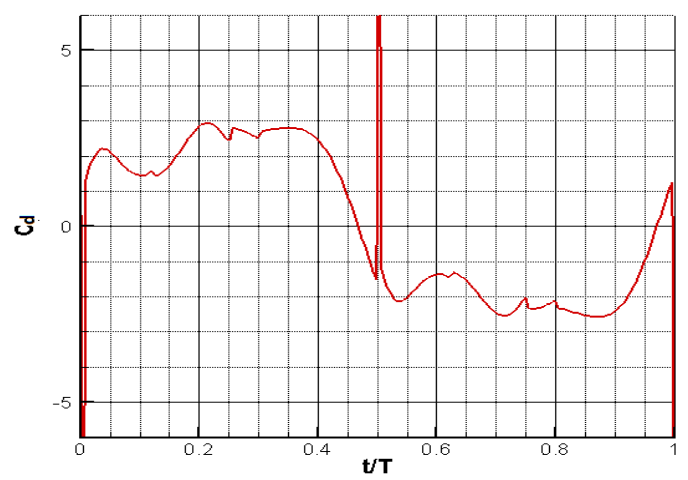

(b) drag coefficients

Figure 12. lift, drag coefficient curve of simplified fruit fly model flapping cycle

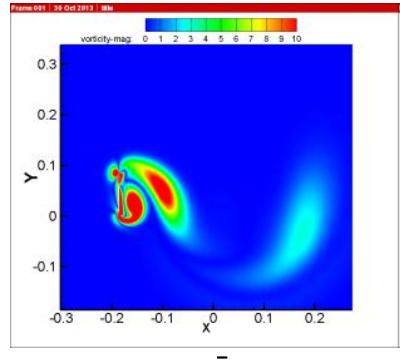

(a) ${ }^{t}=0$

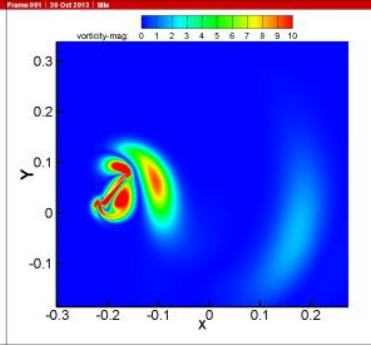

(b) ${ }^{t}=0.05$

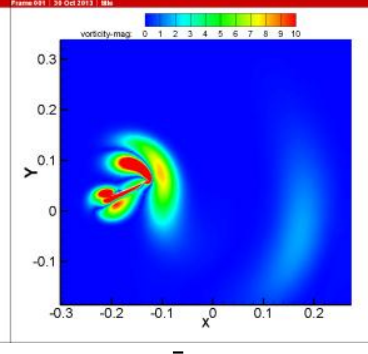

(c) ${ }^{t}=0.1$

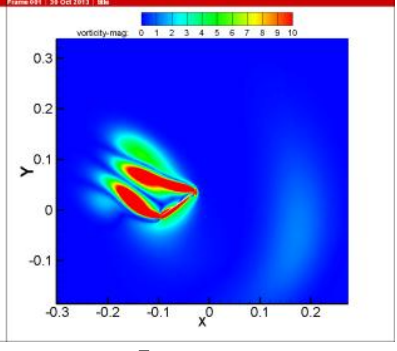

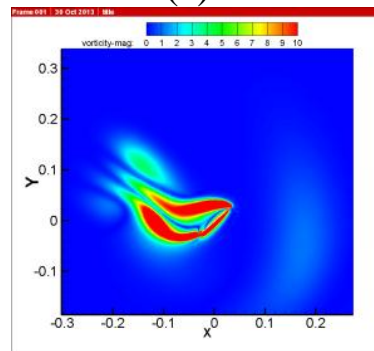

(e) ${ }^{t}=0.25$

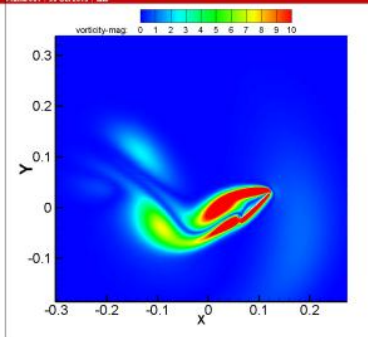

(f) ${ }^{t}=0.325$

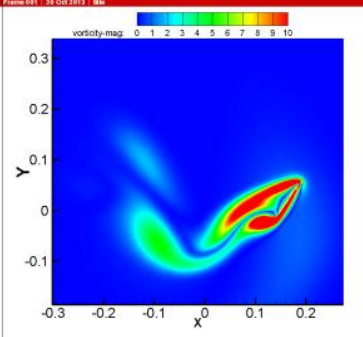

$(\mathrm{g})^{t}=0.4$ $(\mathrm{d})^{t}=0.2$

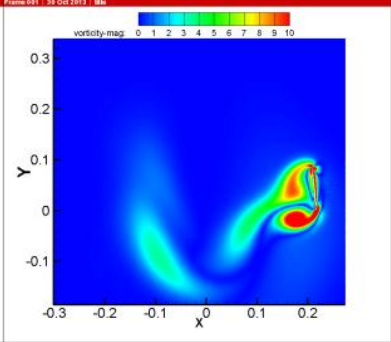

(h) ${ }^{t}=0.5$

Figure 13. Vorticity contour of upward flapping itineraries of the simplified fruit fly model

\section{Conclusions}

In this paper, the flapping wing movement of the hovering fruit flies is simulated. The flapping back and forth motion of the 3D insect wing, upper and lower deviation from the average of the movement and the angle of rotation are simplified to a 2D Airfoil movement. By comparing the lift and drag coefficient curve in both airfoil motion model, the following conclusions:

1. In the harmonic flapping model, the lift curve in a flapping stroke (half of a flapping cycle) has two obvious peak lift at the beginning and end of the stage. The former is caused by the fast acceleration flapping angle, resulting in the rapid rotational force generated by the effect of the 
latter on the back of the airfoil angle of attack. Although the harmonic flapping model can produce a higher lift, its high resistance and low lift-drag ratio lead to a poor aerodynamic performance;

2. The "extra bump" in the curves of the flapping up and down and angle of attack of the simplified model has an important influence on the higher lift. In this model, average drag coefficient is lower, and the average lift coefficient is higher. The ratio of lift to drag is increased roughly by $82.6 \%$ than the harmonic wave flapping model. Although the fruit fly's flapping is more complicated, it is very reasonable, which can produce a better aerodynamic performance.

\section{References}

1. Ellington C P, Van den Berg C, Willmott A P, Thomas A L R. Leading-edge vortices in insect flight [J]. Nature, 1996, 384: 626-630.

2. Mueller T J. The influence of laminar separation and transition on low Reynolds numbers airfoil hysteresis [J]. Aircraft, 1985, 22(9): 763-770.

3. Birch J M, Dickinson M H. Spanwise flow and the attachment of the leading-edge vortex on insect wings [J]. Nature, 2001, 412: 729-733.

4. Sane $\mathrm{S} P$, Dickinson $\mathrm{M} \mathrm{H}$. The aerodynamic effects of wing rotation and a revised quasi-steady model of flapping flight [J]. Expl Biol, 2005, 205: 1087-1096.

5. Sun M, Tang J. Unsteady aerodynamic force generation by a model fruit fly wing in flapping motion [J]. Expl Biol, 2002, 205: 55-70.

6. Dickinson M H, Lehmann F O, Sane S P. Wing rotation and the aerodynamic basis of insect flight [J]. Science, 1999, 284: 1954-1960.

7. Ricardo Galvao, Emily Israeli, Arnold Song, et al. The aerodynamics of compliant membrane wings modeled on mammalian flight mechanics [J]. AIAA 97-1993, 1997.

8. Wang, Z. J., Birch, M. B. \& Dickinson, M. H. Unsteady forces and flows in low Reynolds number hovering flight: two-dimensional computations vs robotic wing experiments [J]. Expl Biol, 2004, 207: 461-474.

9. FRANK M. BOS1, D. LENTINK, B. W. VAN OUDHEUSDEN, H. BIJL. Influence of wing kinematics on aerodynamic performance in hovering insect flight [J]. Fluid Mech, 2008, 594, 341368. 